\title{
An Optimization Model for Antenna Selection and Deployment in Single and Multi-cell RFID Systems
}

\author{
Sicheng Zou ${ }^{*}$, Michael Crisp ${ }^{*}$, Sithamparanathan Sabesan*, Abdullah Kadri ${ }^{+}$, Richard V. Penty ${ }^{*}$ and \\ Ian H. White* \\ "Electrical Division, Department of Engineering \\ University of Cambridge, Cambridge, UK \\ Email: zs271@cam.ac.uk \\ ${ }^{+}$Qatar Mobility Innovations Center \\ (QMIC) \\ Doha, Qatar
}

\begin{abstract}
This paper presents an optimization model for antenna selection and deployment in ceiling mounted, single and multi-cell RFID systems. The proposed model utilises a three-dimensional (3D) antenna radiation pattern, taking into consideration of both antenna half power beam width and downtilt angle. Specifically, we have analysed the effects of cell structures on detection probability in single-cell operation and signal-to-interference ratio (SIR) in multi-cell scenario. Four cell structures have been studied, namely, triangular cells, conventional rectangular cells, offset rectangular cells and hexagonal cells. Several design examples are also presented to show how to use this model to find the optimal configuration to cover the maximum area with a minimum number of antennas for a single cell scenario, as well as the trade-offs that have to be made to achieve a reasonable SIR in multi-cell operation. We believe this optimization model provides new insights into the optimal deployment of single and multi-cell RFID systems.
\end{abstract}

Keywords-Passive radio frequency identification (RFID), multi-cell, $3 D$ modelling, optimization, cell deployment, signal to interference ratio (SIR)

\section{INTRODUCTION}

Radio-frequency identification (RFID) technology has received increasing research interests due to its huge potential in asset tracking and supply chain management. The most promising feature of this technique is its capability to identify an object from a distance, whereas traditional bar-code techniques only allow an item to be detected within a short distance under line-of-sight (LOS) conditions [1]. There are mainly two links in passive RFID systems, namely, forward link and backscattered link. The forward link is to power up passive RFID tags and send inventory commands, while in the backscattered link, tags reflect the reader signal modulated with its unique identifier.

To enable RFID coverage in large areas, such as supermarkets, libraries and airports, multiple readers are required to form a multi-cell RFID system (similar to cellular radio). In such scenarios reader antennas are usually separately mounted on the ceilings and connected to the reader via SMA or TNC cables, instead of being embedded in the RFID portal system as widely used in RFID authentication. However, when multiple readers are running simultaneously there may be performance degradation due to interference. Interference can occur in both the forward link and the backscattered link, causing reader-to-tag interference (R-T interference) and reader-to-reader interference (R-R interference), respectively [2].
Substantial research has been conducted to analyse the interference effect on tag detection [3]-[5] and to solve reader collision problems. R-R interference can be significantly reduced by frequency hopping [6], dense reader mode (DRM) operation [7], or using listen before talk (LBT) [8]. However, these solutions are not able to reject R-T interference since tags have no frequency selectivity [9]. In order to mitigate R-T interference, adaptive power control and probabilistic power control have been proposed to dynamically adjust the output power of dense RFID readers and hence achieve an acceptable forward link signal-to-noise ratio (SIR) [10]. Other alternatives based on time scheduling schemes include round-robin scheduling [11], genetic algorithms and swarm intelligence [12], ARNS-Adaptive RFID Network Scheduling scheme [13] and self-learning hierarchical control nodes [14]. However, these approaches require perfect synchronization among all readers, which is difficult to achieve for a large network consisting of a huge number of readers [9].

The above studies mainly consider using complicated algorithms to mitigate interference effect from adjacent readers. However, few have investigated the effects of antenna selection and cell deployment on tag detection probability and coverage area [15]. In [15] cellular deployment of RFID readers has been studied but only hexagonal cells using a single omnidirectional antenna placed at the centre of each cell are simulated. This is rarely the case in practical deployments since current commercial RFID readers often use directional antennas due to the higher EIRP allowable for antennas with $<90$ degree beamwidth Similarly, the RFID cell planning problem has been studied in [16], which uses a minimum deployment cost function to optimize the antenna positions in a rectangular multi-cell scenario. However, the proposed model is purely mathematical without considering the physical properties such as antenna radiation lobes, tag sensitivity, transmitting power level, etc.

In this paper, we propose a 3D optimization model for antenna selection and cell deployment in a single and multi-cell RFID system. The optimization problem is defined as covering a certain area with a certain probability of detecting a tag at a random location within the area (e.g.,99.9\%) using a minimum number of antennas. By analysing the effects of antenna downtilt angle, half power beamwidth and cell structure on tag detection probability and signal-to-interference ratio (SIR), we can find the optimal antenna selection and cell arrangement strategy based on 


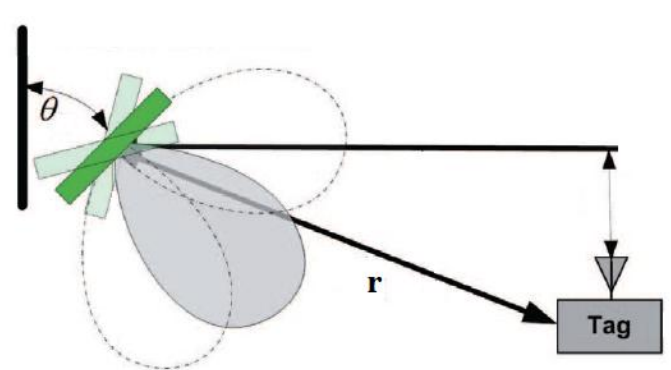

Figure 1Antenna downtilt angle

design specifications. Several design examples have also been presented to demonstrate the feasibility of this model. To the best of our knowledge, this is the first work that provides an optimization strategy based on antenna selection and cell arrangement using modelling of a 3D multi-cell RFID system.

The paper is organized as follows. Section II proposes a comprehensive 3D mathematical modelling of a single and multi-cell RFID system. Simulation results and optimization strategies are presented in Section III. Finally, Section IV concludes the paper.

\section{Modelling OF Single AND Multi-CELl RFID SYSTEM}

\section{A. Modelling of single-cell RFID system}

A reasonable approximation of the $3 \mathrm{D}$ radiation pattern of a single directional antenna can be modelled as:

$$
\begin{gathered}
G_{T x}=\left\{\begin{array}{lr}
G_{m} \operatorname{Real}\left((\cos \varphi \cdot \sin \theta)^{\mathrm{x}}\right) & -\frac{\pi}{2} \leq \varphi \leq \frac{\pi}{2} \\
G_{b} \operatorname{Real}\left((\cos \varphi \cdot \sin \theta)^{\mathrm{x}}\right) & \frac{\pi}{2} \leq \varphi \leq \frac{3 \pi}{2}
\end{array}\right. \\
G_{m}=\frac{35000}{\theta_{b, h} \cdot \theta_{b, v}}
\end{gathered}
$$

where $G_{T x}$ is the gain pattern of the transmitting reader antenna, $G_{m}$ is the maximum gain of the main lobe and $G_{b}$ is the maximum gain of the backlobe, $\varphi$ is the angle between the tag direction and the maximum gain direction of the Tx antenna, and $\theta$ is the downtilt angle of the Tx antenna as shown in Figure 1. $\mathrm{x}$ is a calibration parameter which determines the antenna half power $(3 \mathrm{~dB})$ beamwidth. $\theta_{b, h}$ and $\theta_{b, v}$ are the horizontal and vertical half power beamwidth, respectively. An example of the effects of the half power beamwidth on the radiation lobe is shown in Figure 2. The relationship between $\mathrm{x}$ and the antenna half power beamwidth $\theta_{b}$ is found by scanning $\mathrm{x}$ and calculating its corresponding beamwidth. The obtained curve can then be fitted using the following equation with its parameters shown in Figure 3.

$$
x=a e^{b \theta_{b}}+c e^{d \theta_{b}}
$$

where $a=148.7, b=-0.084, c=15.25, d=-0.023$

It can be seen from the plots that the root mean square error (RMSE) of the fitted equation is only 0.035 and therefore the fitted equation can be used to calculate required the calibration factor $\mathrm{x}$ accurately given a certain beamwidth.

The forward link power (power received by the tag) can be calculated according to Friis eqation.
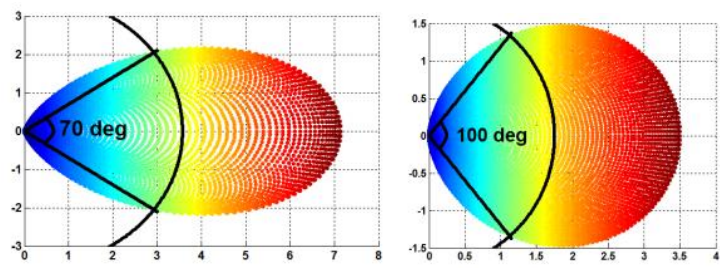

Figure 2 3D model of antenna radiation pattern with half power beamwidth (a) $70^{\circ}$ (b) $100^{\circ}$

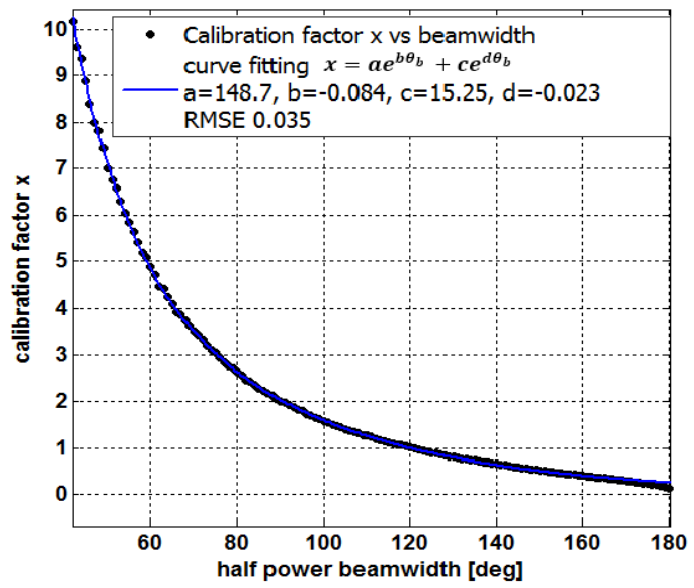

Figure 3 Calibration factor $\mathrm{x}$ vs antenna half power beamwidth

$$
\mathrm{P}_{\mathrm{RX}}=\mathrm{P}_{\mathrm{TX}} \mathrm{G}_{\mathrm{TX}} \mathrm{G}_{\mathrm{tag}}\left(\frac{\lambda}{4 \pi \mathrm{r}}\right)^{2} \cdot \mathrm{PLF}
$$

where $\mathrm{P}_{\mathrm{RX}}$ is the received power of a tag, $\mathrm{P}_{\mathrm{TX}}$ is the transmitted power of the antenna and $G_{\text {tag }}$ is the gain of the tag antenna. $\lambda$ is the wavelength of the transmitted signal and $r$ is the distance between the transmitting antenna and the tag. PLF is polarization loss factor. Consider a Rician fading channel, the received forward link power can then be represented as:

$$
\mathrm{P}_{\mathrm{RX}, \text { rician }}=\left|\sqrt{\mathrm{P}_{\mathrm{RX}}} e^{j 2 \pi \frac{r}{\lambda}}+\sqrt{\frac{\mathrm{P}_{\mathrm{RX}}}{k}} e^{j \varphi_{r}}\right|^{2}
$$

where $\mathrm{k}$ is the Rician factor quantifying the ratio of the power of direct line-of-sight (LOS) and non-LOS components, and $\varphi_{r}$ is a random number ranging from 0 to $2 \pi$, which represents the phase shift of the sum of the non-LOS components.

In a typical passive RFID system the forward link power is usually the factor which limits the tag reading range. Therefore, it is assumed that the reader can read a tag provided that the forward link power delivered to the tag is greater than the tag sensitivity. In addition, since the reader cycles through all Tx antennas one at a time, the forward link power delivered to tags at a specific position within a single-cell RFID system is effectively the maximum forward link power when the reader is cycling through all possible Tx antennas. Hence the forward link power delivered to the tag within the cell can be represented as:

$$
\begin{aligned}
& P_{f}=\max \left(\mathrm{P}_{\mathrm{RX}, \text { rician }}{ }^{i}\right) \\
& i=1,2,3, \ldots N
\end{aligned}
$$

where $i$ represents the $i^{\text {th }}$ transmitting antenna and $N$ is the total number of antennas in each cell. $P_{f}$ is the total forward link power delivered to tags within the cell. The detection 

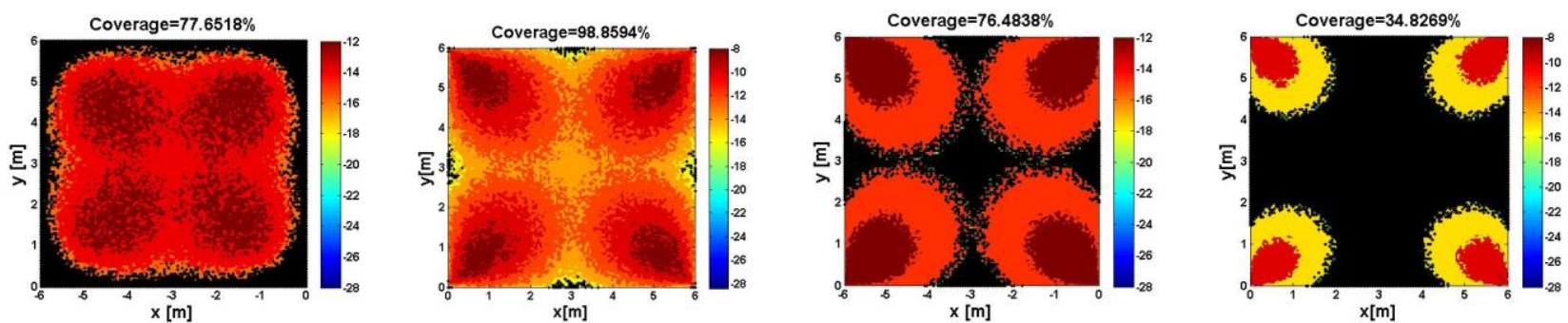

Figure 4 Forward link power distribution of rectangular cell when antenna downtilt angle is (a) $10^{\circ}$

(b) $45^{\circ}$ (c) $70^{\circ}$ (d) $90^{\circ}$

probability can therefore be defined as the area where forward link power is:

$$
P_{D}=\frac{A\left(P_{f}>P_{t h}\right)}{A\left(P_{f}\right)}
$$

where $P_{t h}$ is the sensitivity of the tag, $A\left(P_{f}\right)$ is the area of a single cell and $A\left(P_{f}>P_{t h}\right)$ is the area inside the cell where forward link power is higher than tag sensitivity.

The maximum area of a single cell can be defined as the maximum cell area where $P_{D}$ is above certain reliability (e.g., $99.9 \%$ reliability).

$A_{\max }=\max \left(A\left(\mathrm{P}_{\mathrm{D}}>P_{\text {reliability }}\right)\right)$

Since each cell configuration might have different number of antennas, we also normalize its maximum area by defining the effective coverage per antenna as:

$A_{e f f}=\frac{A_{\max }}{N}$

\section{B. Modelling of multi-cell RFID system}

The signal to interference ratio in a multi-cell RFID system is defined as the ratio of the forward link power delivered to the desired cell to that which comes from other neighboring cells. Since the signals from each interfering cell are incoherent, the total power of the interference signal can be regarded as the scalar sum of the power leakage into the desired cell from each neighboring cell. However, since the reader in each cell keeps cycling through all $\mathrm{Tx}$ antennas, the forward link power from the desired cell and the interfering cell will vary. Consider the best case when the interference signal from

\begin{tabular}{|l|l|}
\hline Antenna type & Circularly polarized \\
\hline $\begin{array}{l}\text { Antenna half power } \\
\text { beamwidth }\end{array}$ & $70^{\circ}$ \\
\hline Antenna maximum gain & $8.5 \mathrm{dBi}$ \\
\hline Front to back ratio & $12 \mathrm{~dB}$ \\
\hline Polarization loss factor & 0.5 \\
\hline Tx power & $35 \mathrm{dBm}$ EIRP \\
\hline Rician K factor & 100 \\
\hline Antenna plane & $\begin{array}{l}3 \mathrm{~m}, 3.5 \mathrm{~m} \text { and } 4 \mathrm{~m} \\
\text { above ground }\end{array}$ \\
\hline Tag plane & $1 \mathrm{~m}$ above ground \\
\hline Tag sensitivity & $-15 \mathrm{dBm}$ \\
\hline Cell configuration & $6 \mathrm{~m} * 6 \mathrm{~m}$ Rectangle \\
\hline Num of antennas & 4 \\
\hline
\end{tabular}

Table 1 Simulation parameters neighboring cells causes minimum interference at a particular location within the desired cell, the total interference power can then be represented as:

$$
\begin{gathered}
P_{I, \text { best }}=\sum_{k=1}^{m} \min \left(\mathrm{P}_{\mathrm{RX}, \text { rician }, \mathrm{k}}^{i}\right) \\
i=1,2,3, \ldots N
\end{gathered}
$$

where $m$ is the number of interfering cells, $\mathrm{P}_{\mathrm{RX}, \text { rician,k }}$ is the forward link power leaked into the desired cell from $k^{\text {th }}$ interfering cell. Similarly, the worst case scenario is when all the interfering cells happen to cause maximum interference at a particular location within the desired cell and the total interference power in this case can be represented as:

$$
P_{I, \text { worst }}=\sum_{k=1}^{m} \max \left(\mathrm{P}_{\mathrm{RX}, \text { rician, } \mathrm{k}}^{i}\right)
$$

For simplicity, we assume the average interference is the mean value between the best case and the worst case and hence the average interference power to the desired cell is:

$P_{I, \text { ave }}=\frac{P_{I, \text { best }}+P_{I, \text { worst }}}{2}$

As illustrated in II.A, the forward link power delivered to the desired cell can be represented as:

$$
\begin{gathered}
P_{f, \text { desired }}=\max \left(\mathrm{P}_{\mathrm{RX}, \text { rician, desired }}{ }^{i}\right) \\
i=1,2,3, \ldots N
\end{gathered}
$$

Therefore, the average signal to interference ratio in the desired cell is denoted as:

$S I R=\frac{P_{f, \text { desired }}}{P_{I, \text { ave }}}$

In a similar manner to the case of the power distribution in a single-cell, the SIR in the desired cell will also vary with tag position. To indicate the overall interference level we can use the average SIR in the desire cell:

$S I R_{\text {ave }}=\overline{S I R}$

The average SIR can then be used to measure the optimality of a cell arrangement in multi-cell operation.

\section{RESULTS AND DISCUSSIONS}

\section{A. Effects of downtilt angle on detection probability}

To investigate the impact of antenna downtilt angle (defined as the angle between the horizontal plane and the maximum gain direction of the antenna), we consider a rectangular cell where one antenna is placed at each corner. 


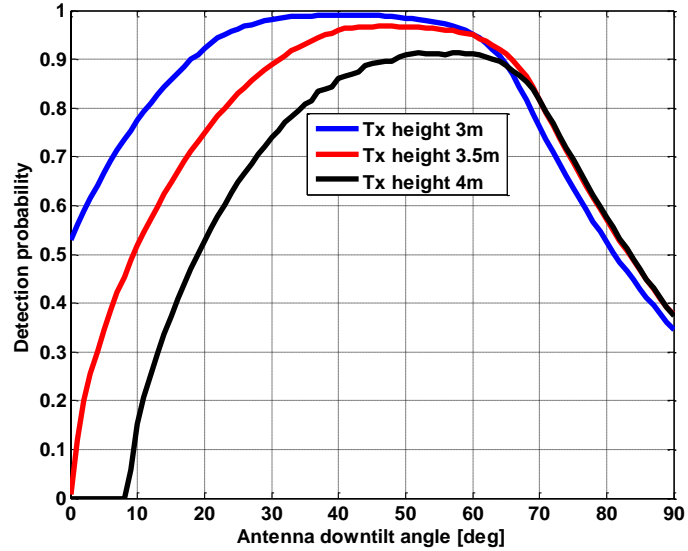

Figure 5: Optimal downtilt angle for rectangular cell

The detailed antenna and cell parameters are presented in Table I. The tag sensitivity of current UHF Gen2 RFID chips has been reported to range from -11 to $-18 \mathrm{dBm}$. Therefore it is assumed that tag sensitivity is $-15 \mathrm{dBm}$ in the following simulation. The forward link power distribution for this single-cell can then be obtained from (6) and its detection probability can be calculated according to (7). Figure 4 shows the power distribution of the rectangle for different downtilt angles. The area where forward link power is below tag sensitivity is shaded in black. It is clear that downtilt angle has a significant effect on tag detection probability. A downtilt angle of $45^{\circ}$ results in a much better detection probability compared to that in the case of $70^{\circ}$ or $90^{\circ}$. In addition, it is noted that a larger downtilt angle results in higher power projected to the tag plane closer to the antenna positions, although the total reading range is reduced because most areas are not covered by antenna main radiation lobe.

Next, we evaluate the variation of the tag detection probability with respect to antenna downtilt angle as illustrated in Figure 5. As can be seen from the figure, the optimal downtilt angles are $45^{\circ}, 50^{\circ}$ and $55^{\circ}$ when the antenna height is $3 \mathrm{~m}, 3.5 \mathrm{~m}$ and $5 \mathrm{~m}$, respectively. Another interesting result is that when the downtilt angle is $90^{\circ}$, the detection probability slightly increases when the antenna is raised from $3 \mathrm{~m}$ to $4 \mathrm{~m}$, instead of dropping significantly as one would expect. This is because when an antenna is raised

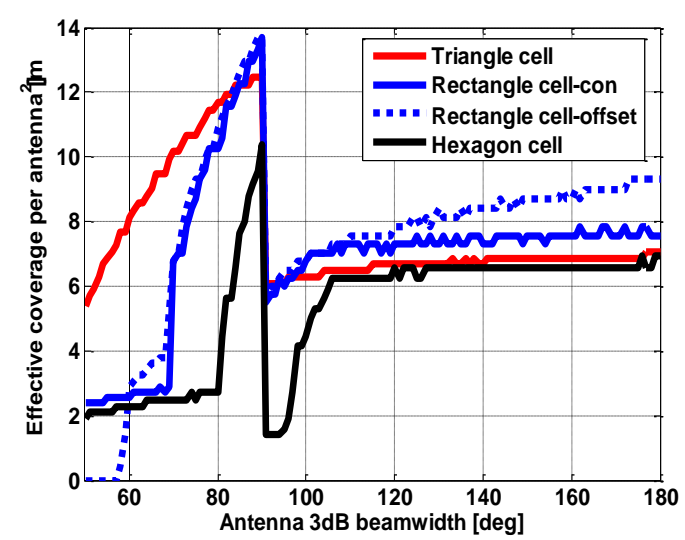

Figure 8 Effective coverage per antenna vs antenna half power beamwidth

to a higher plane, the tag plane will be better aligned with antenna main radiation lobe (smaller deviation from the main lobe), and hence a larger effective gain. This compensates for the power loss introduced when Tx antenna is moved further away from the tag plane and therefore a similar detection probability is seen although antennas have been raised by $1 \mathrm{~m}$.

\section{B. Optimization of single-cell RFID systems}

In this section we analyse the performance of four cell configurations, namely, triangular cell, hexagonal cell, conventional rectangular cell and offset rectangular cell. In the first three configurations an antenna is placed at each corner facing towards the cell centre, whereas in the last configuration only two antennas are at the corners while the other two are placed at the centre of two edges. The above cell configurations are illustrated in Figure 6, where the black arrows represent the antenna positions and their facing directions. The power distribution of these four cell configurations are calculated from (6) and illustrated in Figure 7 assuming the antenna half power beamwidth is $70^{\circ}$.

In order to evaluate the performance of each cell configuration, we compare their effective coverage area per antenna according to (8) and (9), where $P_{\text {reliability }}$ is set to $99.9 \%$ and the downtilt angle is set to $45^{\circ}$. The other parameters are the same as those defined in Table I. To investigate how effective coverage per antenna will be
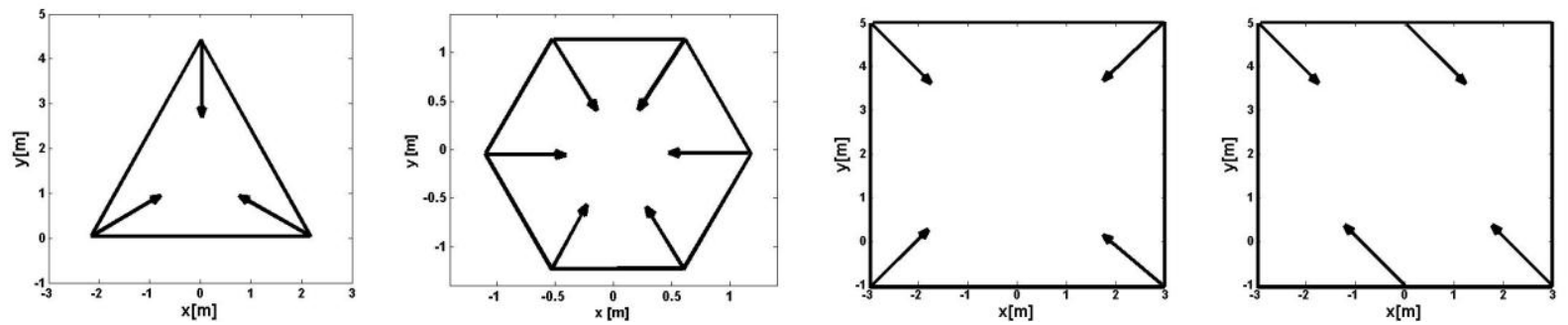

Figure 6 Antenna positions and directions for: (a) Triangular cell (b) Hexagonal cell (c) Conventional rectangular cell (d) Offset rectangular cell
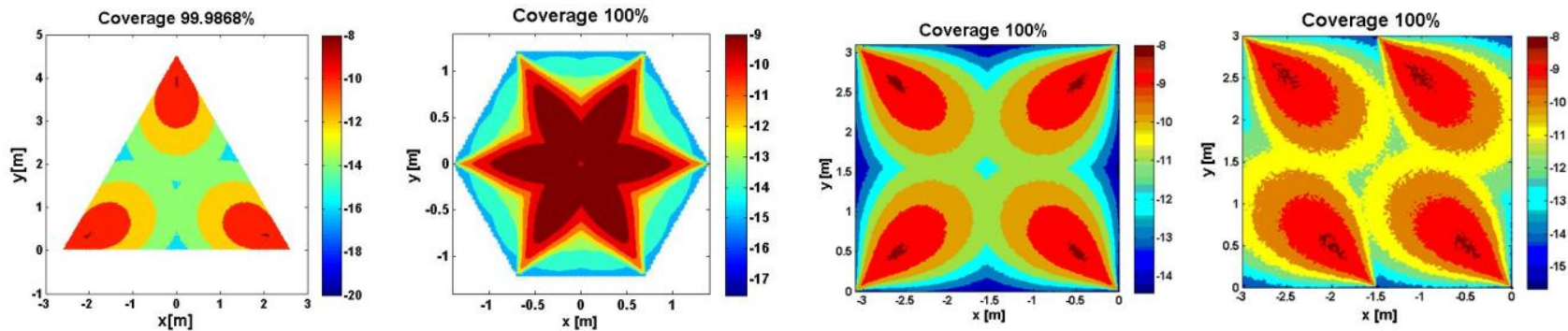

Figure 7 Forward link power distribution of four cell configuration in $\mathrm{dBm}$ (a) Triangular cell (b) Hexagonal cell (c) Conventional rectangular cell (d) Offset rectangular cell 
affected by antenna half power beamwidth, we plotted the effective coverage per antenna of all four cell configurations with respect to antenna half power beamwidth. As shown in Figure 8 , when antenna beamwidth is small (from $50^{\circ}$ to $80^{\circ}$ ), the triangular cell has the maximum effective coverage per antenna compared with the other three cell configurations. This is because of the geometric feature of triangles, which only requires a smaller antenna beamwidth to effectively cover the whole triangular area. Conventional rectangular cells and offset rectangular cells perform similarly in this range, whereas hexagonal cells have the minimum effective coverage per antenna at all beamwidths. It is also noted that there is an abrupt drop of effective coverage when the antenna beamwidth reaches $90^{\circ}$. This is due to current regulation which limits the equivalent isotropic radiation power (EIRP) of an antenna to be $3 \mathrm{~dB}$ down when its beamwidth is above $90^{\circ}$. Beyond $90^{\circ}$ the offset rectangular cell seems to outperform the other three configurations. A possible reason is that the offset rectangular configuration aims at minimising overlap among antenna radiation lobes to cover a large area, and a larger beamwidth might make it more effective.

\section{Design examples in optimising single-cell RFID}

In order to demonstrate how to use this model to optimize a single-cell RFID system, we proposed a design example

\section{Question:}

Antennas are mounted at $3 m$ high ceilings with $45^{\circ}$ downtilt angle and targeted tag plane is $1 \mathrm{~m}$. If we are able to choose any antenna types, which cell configuration can cover a maximum area with minimum number of antennas and what is the maximum area that it can cover with more $99.9 \%$ detection probability?

\section{Solutions:}

According to Figure 8, the maximum effective coverage per antenna occurs when the conventional rectangular or offset rectangular cell is used with a $90^{\circ}$ antenna half power beamwidth. Hence we can select either a conventional rectangular or an offset rectangular cell as the optimal cell configuration in this case. The maximum cell area that it can cover can be calculated as $13.7 \mathrm{~m}^{2} * 4=54.8 \mathrm{~m}^{2}$. Therefore, according to the area formula for a rectangular cell $\mathrm{A}=a^{2}$ the maximum length of each side of the rectangle $a$ is $7.4 \mathrm{~m}$.

\section{Optimization of Multi-cell RFID system}

To analyse how the above cell configurations perform in a multi-cell scenario, we consider the interfering signal from all neighbouring cells as shown in Figure 9. DC represents the desired cell and IC represent interfering cell. The triangular cell pattern has 12 neighbouring cells, the rectangular cell pattern has 8 neighbouring cells, and

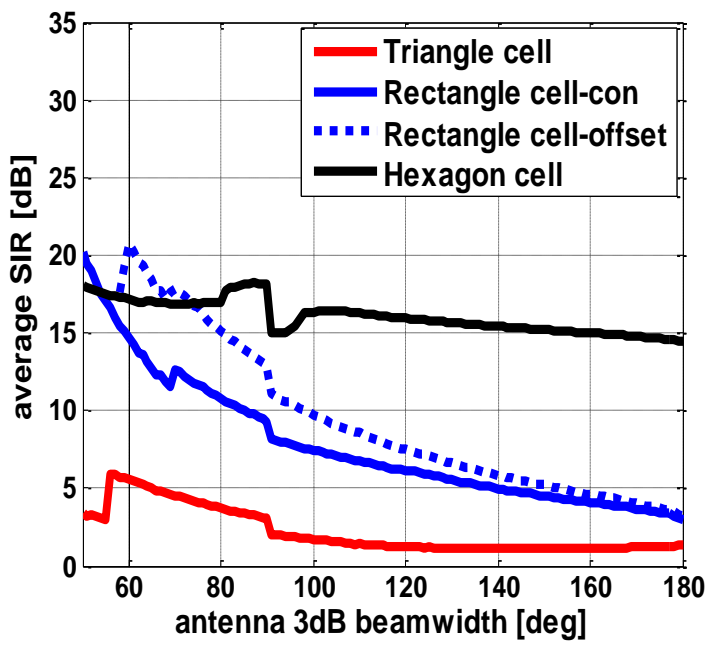

Figure 10 Average SIR vs antenna half power beamwidth

hexagonal cell pattern has 6 neighbouring cells.

It is assumed that cells beyond nearest neighbours will produce negligible interference on the desired cell due to increasing path loss with distance. The average SIR of the cell configurations can then be calculated according to (15). The simulation parameters are the same as those used in Section III except that the simulated cell area is the maximum area that it can cover with more than $99.9 \%$ detection probability according to the results in Figure 8. Figure 10 illustrates how the average SIR of the above cell configurations varies with antenna half power beamwidth. The results show that in general the average SIR of all cell configurations decreases as antenna half power beamwidth increases, although at some points there is a slight variation in the average SIR. This is expected since a larger beamwidth can result in more power leakage into the desired cell and hence the SIR will be reduced. It is also noted that when the antenna half power beamwidth is relatively small (from $55^{\circ}$ to $75^{\circ}$ ), the offset rectangular cell has the best SIR, whereas when it is above $75^{\circ}$, the average SIR of hexagonal cell is significantly better compared with the other three configurations. The triangular cell, on the other hand, has the worst SIR regardless of the antenna half power beamwidth. This is because triangular cell has the largest number of neighbouring cells and interfering antennas (5 neighbouring interfering antennas compared to the 3 neighbouring interfering antennas in the conventional rectangular cell, 2 neighbouring interfering antennas in the hexagon cell and 1 neighbouring interfering antenna in offset rectangular cell).

\section{Design example in optimising multi-cell RFID}

Similar to Section III, we also propose a design example to illustrate how to design cell arrangement for multi-cell RFID
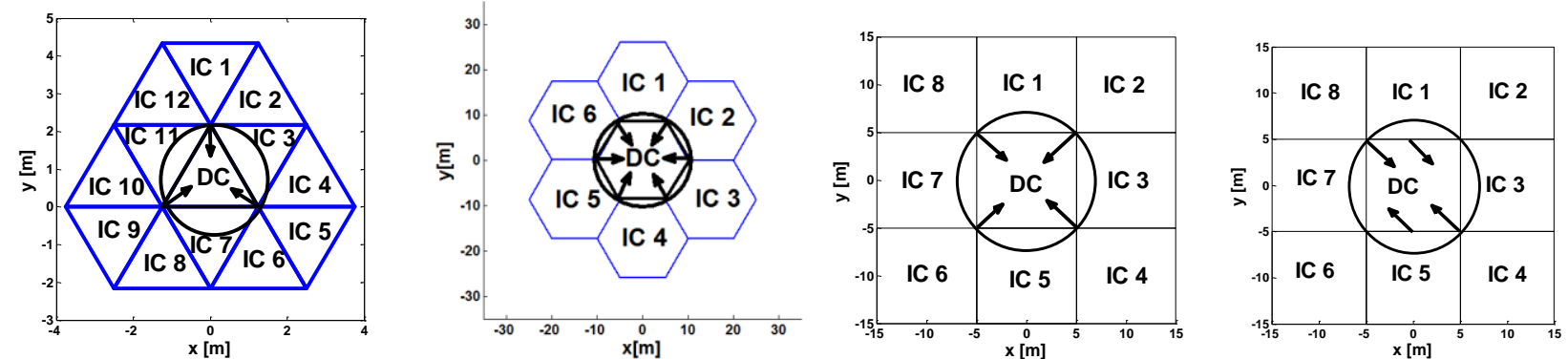

Figure 9 Four multi-cell configurations (a) Triangular multi-cell (b) hexagon multi-cell (c) conventional rectangular multi-cell (d) offset rectangular multi-cell 
systems based on design specifications.

\section{Question:}

Antennas are mounted at $3 m$ high ceilings with $45^{\circ}$ downtilt angle and targeted tag plane is $1 \mathrm{~m}$. If available antennas have $80^{\circ}$ half power beamwidth and targeted average SIR is $15 \mathrm{~dB}$, which cell configuration can cover a maximum area with minimum number of antennas and what is the maximum area that it can cover with more $99.9 \%$ detection probability?

\section{Solutions:}

According to Figure 10, when the antenna half power beamwidth is $80^{\circ}$, the offset rectangular cell and the hexagon cell are the only two cell configurations with average SIR more than $15 \mathrm{~dB}$. Therefore, we can select these two cell configurations as potential candidates. We then go back to Figure 8 . At $80^{\circ}$ beamwidth the offset rectangular cell has a better effective coverage per antenna $\left(10 \mathrm{~m}^{2}\right)$, and hence we can select this cell arrangement and the maximum area that it can cover with more $99.9 \%$ detection probability is $10 \mathrm{~m}^{2} *$ $4=40 \mathrm{~m}^{2}$. Therefore the maximum length of each side of the rectangle is $\sqrt{40}=6.3 \mathrm{~m}$.

\section{Guide for practitioner}

It should be noted that in order to use the above optimization model in a realistic scenario, the calibration of loss factor is required since the loss factor in a realistic scenario can be different from the parameters used in simulation due to impedance mismatch or multipath fading. To calibrate the loss factor one can move a tag in the maximum gain direction of the reader antenna until it cannot be detected with a certain detection rate (e.g. 99\%). The measured maximum range can then be used to calibrate the loss factor used in the simulation.

\section{CONCLUSIONS}

In this paper, we have proposed an optimization model for antenna selection and deployment in single and multi-cell RFID systems. A 3D analytical modelling of a single and multi-cell RFID system is presented with adjustable antenna beamwidth, downtilt angle and cell arrangement. It is found that the detection probability of a single-cell RFID system is highly sensitive to downtilt, and the optimal downtilt angle can vary with antenna height and tag height above the ground. In addition, we have compared the effective coverage per antenna of four possible cell arrangements in a single-cell scenario. It is found that triangular cell can cover the maximum area with a minimum number of antennas when the antenna beamwidth is small while an offset rectangular cell can cover the largest area at larger beamwidths. In contrast, when the above cell configurations form a multi-cell system, triangular cells have the worst average forward link SIR at all beamwidths while offset rectangular cells and hexagonal cells have the best SIR at small and large beamwidths, respectively. Therefore, there is a trade-off between maximising coverage area and achieving a reasonable SIR in multi-cell application. Finally, we have demonstrated the feasibility of the proposed optimization model through several design examples and provided guidance for practitioners. We believe that this model might be a useful tool in optimal antenna selection and cell deployment in a multi-cell RFID system.
This work was made possible by NPRP grant \#NPRP4-7262- 272 from the Qatar National Research Fund (a member of The Qatar Foundation). The statements made herein are solely the responsibility of the authors

\section{REFERENCES}

[1]. R. Want, "An introduction to RFID Technology," Pervasive Computing, IEEE, vol.5, no.1, pp.25,33, Jan.-March 2006

[2]. D.W. Engels, S. E. Sanjay, "The Reader collision problem ," IEEE International Conference on Systems, Man and Cybernetics, vol.3, pp.6-9 Oct. 2002

[3]. D.-Y. Kim, H.-G. Yoon, B.-J. Jang, and J.-G. Yook, "Effects of reader interference on the RFID interrogation range," IEEE Trans. Ind. Electron. vol. 56, no. 7, pp. 2337-2346, Jul. 2009

[4]. A. Lazaro, D. Girbau, and R. Villarino, "Effects of interferences in UHF RFID systems," Progress In Electromagnetics Research, Vol. 98, 425-443, 2009.

[5]. S. Zou; M. Crisp, S. Sabesan, A. Bekkali, A. Kadri, R.V. Penty, I.H. White, , "Tag read enhancement using unsynchronized signal from adjacent readers in multi-cell RFID system," RFID Technology and Applications Conference (RFID-TA), 2014 IEEE, vol., no., pp.62,67, 8-9 Sept. 2014

[6]. FCC Part 15 Radio frequency devices, Section 15.247, Dec. 2003.

[7]. ETSI EN 302 208-1 V1.1.2: Electromagnetic compatibility and Radio spectrum Matters (ERM); Radio Frequency Identification Equipment operating in the band $865 \mathrm{MHz}$ to $868 \mathrm{MHz}$ with power levels up to $2 \mathrm{~W}$; Part 1: Technical requirements and methods of measurement, Jul. 2006. Sdf

[8]. ARIB STD-T89 Ver.2.0: 950MHz-band RFID equipment for premises radio station, Mar. 2006. Sdfasf

[9]. Y. Tanaka and I. Sasase, "Interference Reduction Scheme for UHF Passive RFID Systems Using Modulation Index Control," Proceedings of 14th Asia-Pacific Conference on Communications (APCC'08), pp. 1-5, October 14-16 2008

[10]. K. Cha, A. Ramachandran, and S. Jagannathan, "Adaptive and Probabilistic Power Control Algorithms for Dense RFID Reader Network," Proceedings of the IEEE International Conference on Networking, Sensing and Control (ICNSC '06), pp. $474-479$, 2006. Asdfasf

[11]. K. Finkenzeller, RFID Handbook, Second edition. Jhon Wiley \&Son's, 2003 Asdfasfas

[12]. Chiu Chui-Yu, Ke Cheng-Hsin and K.Y Chen, "Optimal RFID networks scheduling using genetic algorithm and swarm intelligence," Systems, Man and Cybernetics, 2009. SMC 2009. IEEE International Conference on , vol., no., pp.1201,1208, 11-14 Oct. 2009

[13]. Lijun Sun; Shijie Zhou; Jiaqing Luo; Yuehan Zhang; Mengjie Zhang; Weiwei Deng, "ARNS: Adaptive RFID Network Scheduling for Device-Free Tracking," Mobile Ad-hoc and Sensor Networks (MSN), 2013 IEEE Ninth International Conference on , vol., no., pp.248,253, 11-13 Dec. 2013

[14]. J. K. Ho, "Solving the reader collision problem with a hierarchical Q-learning algorithm," Master's thesis, Massachusetts Institute of Technology, Feb. 2003.

[15]. Junhui Zhao; Xuexue Zhang; Shenchun Guan; Wei Yang, "Reader cellular deployment for RFID system," Communications Technology and Applications, 2009. ICCTA '09. IEEE International Conference on, vol., no., pp.44,48, 16-18 Oct. 2009

[16]. Taehoon Kim; Junghwa Shin; Sungwoo Tak, "Cell Planning for Indoor Object Tracking Based on RFID," Mobile Data Management: Systems, Services and Middleware, 2009. MDM '09. Tenth International Conference on , vol., no., pp.709,713, 18-20 May 2009 